\title{
Microcontroller based in vitro hematocrit measurement system
}

\author{
Sinan S. Mohammed Sheet, Mohammed S. Jarjees \\ Technical Engineering College, Northern Technical University (NTU), Mosul, Iraq
}

\begin{tabular}{l}
\hline Article Info \\
\hline Article history: \\
Received Jul 19, 2019 \\
Revised Oct 20, 2019 \\
Accepted Nov 3, 2019 \\
\hline
\end{tabular}

\section{Keywords:}

Hematocrit

Hemoglobin

Microcontroller

Packed cell volume

\begin{abstract}
The hematocrit (HCT) is the most important measurement in the blood profile. It has been used for early diagnose of the specific blood diseases such as anaemia, leukaemia and malaria. The microhematocrit is the conventional method of measurement of HCT manually which is timeconsuming and uncertain due to human error. An automated system for measuring hematocrit will minimize the human-error and the time which will give the ability for medical staff to serve more patients. This paper aims to demonstrate an automated system for measuring the HCT based on microcontroller. The designed system based on Arduino Atmega 2560 microcontroller and combination array of lighting emitting diode and photodetectors. The transmission and the absorption characteristics of the red light $(660 \mathrm{~nm})$ through the centrifuged blood sample in a capillary tube are calculated and used to determine the HCT. The outputs are analyzed to determine the haemoglobin (HB) and packed cell volume (PCV). The significant correlation $(\mathrm{r}=0.9856, \mathrm{p}=3.106 * 10-4)$ between the PCV readings of the proposed system and the conventional method has been observed. The most important finding is the precise of PCV and $\mathrm{HB}$ readings for the proposed system compared with previous automated methods as well as the conventional method have been obtained.
\end{abstract}

Copyright $\odot 2020$ Institute of Advanced Engineering and Science. All rights reserved.

\section{Corresponding Author:}

Mohammed Sabah Jarjees,

Technical Engineering College of Mosul, Nothern Technical University

Al-Jammi'a Street, Main Campus of Northern Technical University, Mosul, IRAQ.

Email: mohammed.s.jarjees@gmail.com

\section{INTRODUCTION}

Hematologic disorder is one of the most prevalent diseases worldwide particularly in the poorest healthcare service countries [1-3]. Anemia, Leukemia and malaria are closely similar parallels of the hematologic disorder [3-5]. The early detection of these diseases helps to apply a suitable treatment and to save the patient's life. The hematocrit (HCT), which is a procedure to determine the packed cell volume (PCV), has been used for early detection of the hematologic disorders. The conventional method to measure HCT is a microhematocrit [6-7]. Although, the microhematocrit has been used to measure the HCT, it has several disadvantages such as: tediousness, time consumption and inaccuracy due to the serendipitousness nature of humans [8]. Therefore, an automated system for measuring HCT has been proposed.

Numerous systems are designed to measure the HCT automatically. The microscopic images of blood sample, for example, has been used to calculate the total number of red and white blood cells [9-13]. The red blood cells (RBCs) have been used to calculate the level of HCT and to measure the Hemoglobin [14-15]. Supriyanti et al. designed and evaluated a computerized diagnosis system to identify the leukocytes in microscopic blood sample images [12]. Moreover, study by Kim et al proposed a physiometer based on microfluidic system to measure the HCT and RBCs on a chip [16]. In this study, the electrical component of the physiometer was used to estimate the hematocrit and the RBC. A computational method has also been used to calculate the RBC in the blood sample [7, 17-18]. This method was based on the segmentation of the microscopic blood imagery using image processing algorithm. 
A microcontroller based system has widely used in industrial and medical applications [19]. It has been used to design an automated system for determining the PCV level. Study by Baban and Yaseen has been designed and implemented a PCV measurement system using Arduino microcontroller [20]. They used the light emitted diodes and photo-detectors with the aid of the microcontroller to estimate the PCV level. However, in this study, there are no results presented to validate the implemented system. Furthermore, they did not take in their accounts the interference and the scattering of the light emitted by diodes (LED). This may lead to inaccurate estimation of the PCV value, HB and HCT levels.

To overcome the weakness in the previous studies, this study gives a new HCT reader design through optimizing the distance between the LEDs and photocells. Thus, isolator slides are used to prevent overlapping among LEDs. An isolated material is also used to enhance streaming and reduce light deviation. Additionally, the evaluation and validation of the proposed system have been approved by comparing the results of the automated (proposed) and the conventional systems.

\section{RESEARCH METHOD}

Three main parts have been focused in this study: Firstly, design and implementation of an automated system for measuring the HCT of the centrifuged blood sample in a capillary tube based on microcontroller. Secondly, in order to validate the proposed system, the results of the HCT, which were obtained from the automated system, have been compared to the results of the convolutional method. Finally, the levels of the PCV and HB have been calculated based on the values of the HCT.

\subsection{Preparing of the Blood Sample}

The hematocrit procedure depends on measurement the percentage of the volume of RBCs to the volume of whole blood in the capillary tube [21-22]. The procedure of preparing the blood sample is started by centrifuged the sample for five minutes at $10,000 \mathrm{rpm}$, this will lead to separate the sample into multilayers: the first layer is the plasma, the second layer is the withe blood cell (WBCs) which is the separate border between the plasma and the third layer which is the RBCs. The RBCs are settled in the bottom of the capillary tube. The multilayer of the centrifuged blood sample in a capillary tube is demonstrated in Figure 1.

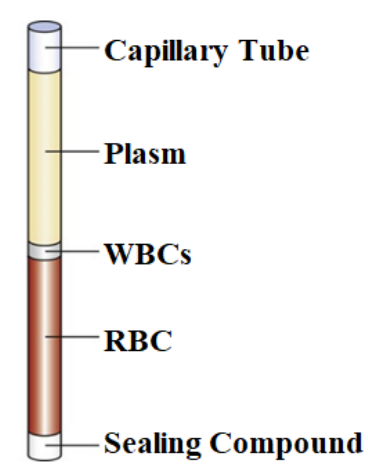

Figure 1. The multilayer of the centrifuged blood sample in a capillary tube

\subsection{The Conventional Method of Measurement the HCT}

The conventional method of measurement the HCT is known as the microhematocrit. The microhematocrit method is recommended by the National Committee for Clinical Laboratory Standards (NCCLS) for evaluating the HCT or PCV level of the centrifuged blood sample [23-24]. The HCT or the $\mathrm{PCV}$ is measured by determining the ratio of RBCs volume to the whole blood volume. This is achieved by measuring the height of RBCs layer and the whole blood sample volume using the microhematocrit reader in

Figure 2 which is expressed as a decimal number or percentage fraction. The mathematical equations which are used to calculate the PVC level and the level of the HB concentration are shown in (1) and (2), respectively [20,23].

$$
\begin{aligned}
& P C V \%=\frac{\text { The height of RBCs layer }}{\text { The height of the whole volume of the blood sample }} \times 100 \\
& H B=\frac{P C V-1}{3}
\end{aligned}
$$




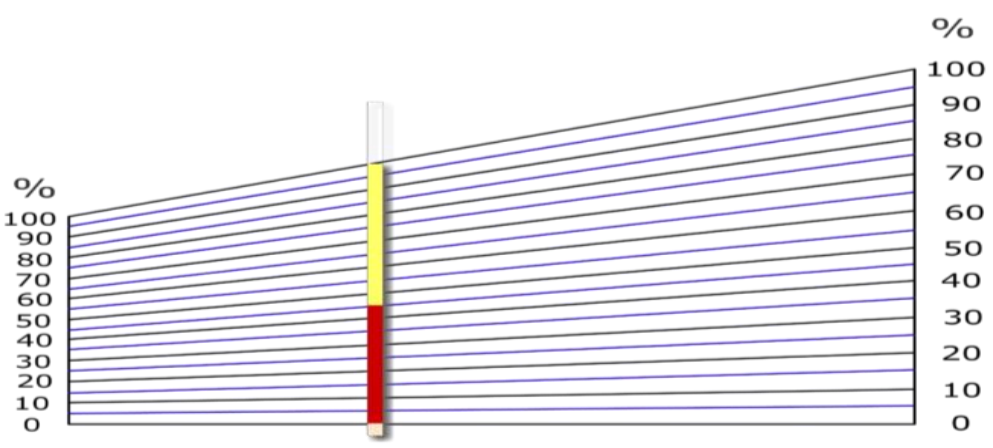

Figure 2. The microhematocrit reader

\subsection{The Proposed Automated Method of Measurement the HCT}

The proposed automated method composed of array of fifteen light emitted diodes (LEDs, wavelength $600 \mathrm{~nm}$ ). An optical isolated material is used to separate the LEDs in order to prevent the light interfacing among the LEDs and to ensure that the specific diode will activate the corresponding photocell. Figure 3 shows the configuration of the LEDs and photocells detectors with isolated materials. The centrifuged blood sample in a capillary tube will put between the LEDs and the photocell detectors. Due to the transparent characteristics of the plasma and the redness of the RBCs, the RBCs absorb the emitted light $(600 \mathrm{~nm})$; they prevent it from passing through and activate the photocells at the level of the RBCs. In contrast, the plasma allows the red light to pass through and to activate the corresponding photocells at the level of plasma. Therefore, the signals that are generated by the photocells can indicate the level of the RBCs and the plasma as well as the whole volume of the blood in the capillary tube. Then, these signals generated by the detectors will send to the microcontroller in order to calculate the PCV and HB levels and to display the results on a screen.

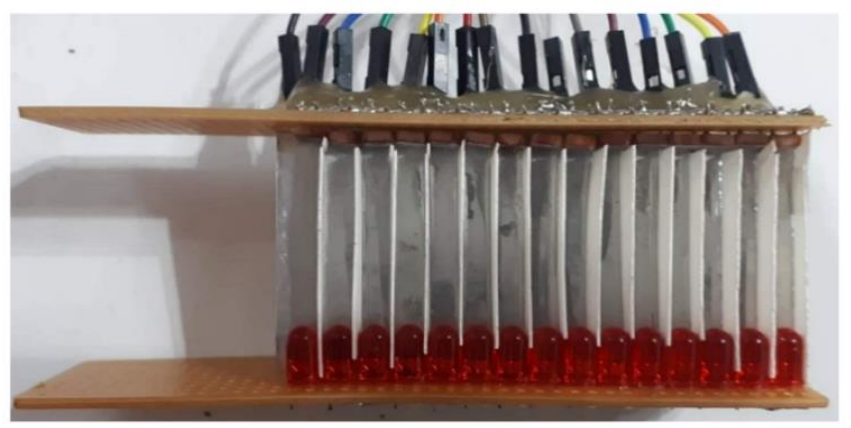

Figure 3. The configuration of the LEDs and photocells detectors with isolated material

\subsection{Arduino Atmega 2560 Microcontroller}

The Arduino Atmega 2560 microcontroller [25] is used in the projected system. This type of microcontroller is considered as user-friendly and inexpensive microcontroller. It comes with a considerable memory space and has more inputloutput pins comparing with the other models of Arduino microcontrollers. It has 54 digital inputloutput pins as well as 16 pins for analog inputs [25]. The Arduino Atmega 2560 microcontroller has a crystal oscillator of $16 \mathrm{MHz}$ built-in the based board and DC power jack. These specifications make this microcontroller suitable for this project.

Figure 4 shows the Arduino Atmega 2560 microcontroller. The flow chart diagram of the operating program for the microcontroller is shown in Figure 5. The signals, which are received from the detectors, will be processed by microcontroller using (1) and (2) in order to determine the level of PCV and HB, respectively. Then, the determined values will be sent to the LCD for displaying. The schematic diagram of the proposed system is shown in Figure 6. 


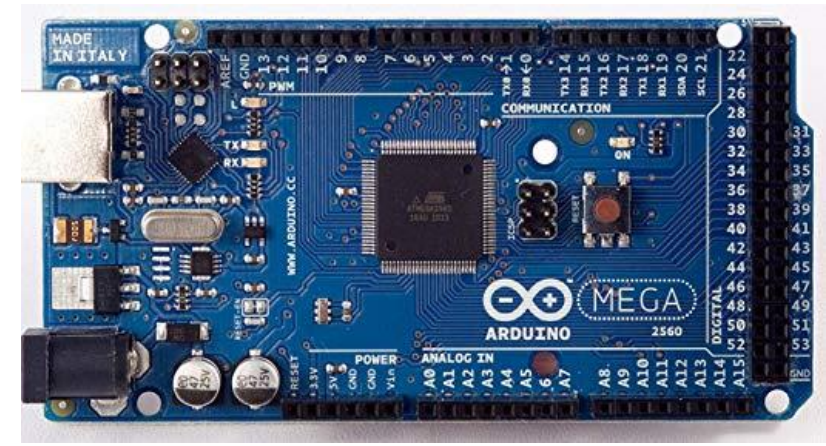

Figure 4. the Arduino Atmega 2560 microcontroller

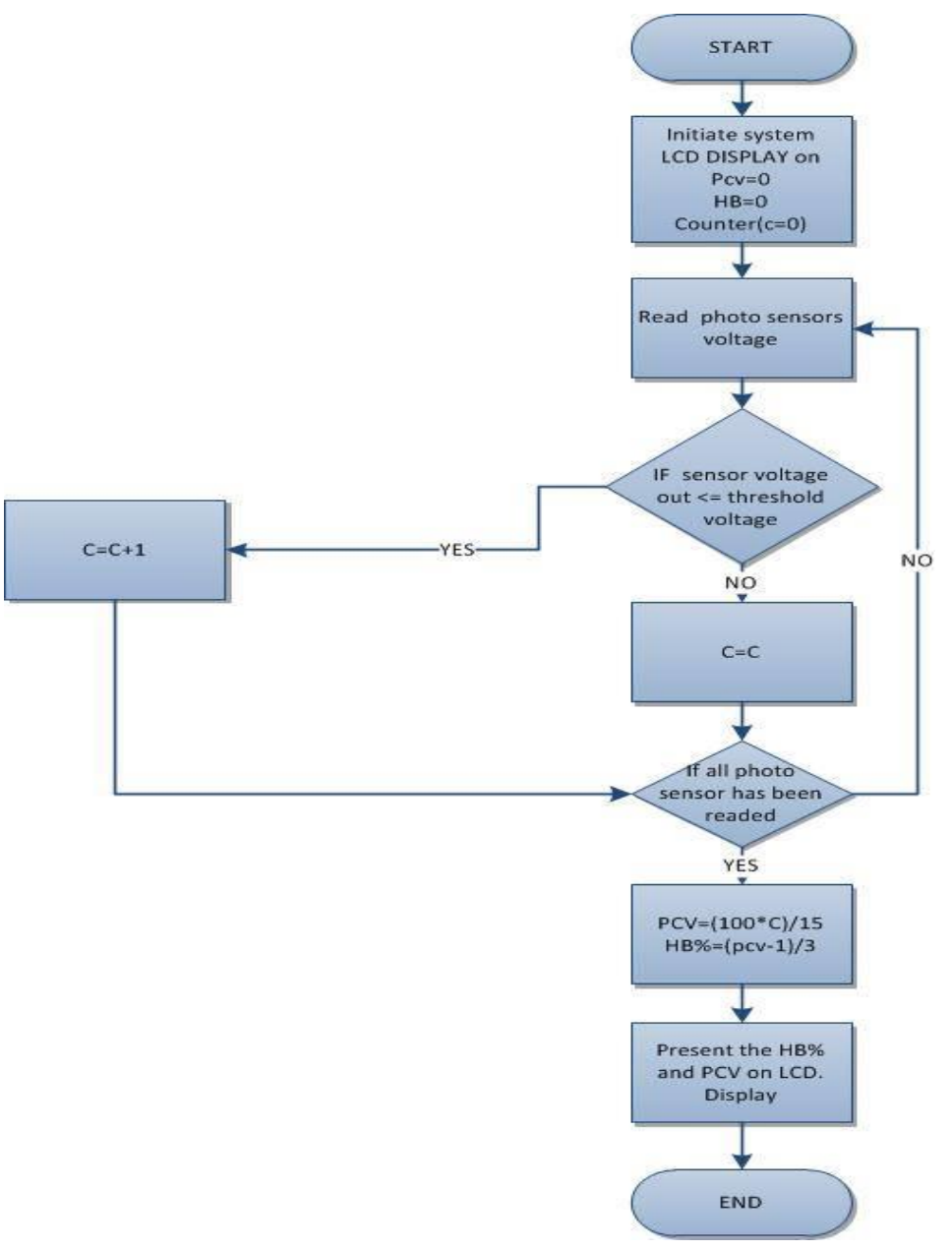

Figure 5. The flow chart diagram of the operating program for the microcontroller

\section{RESULTS AND ANALYSIS}

The proposed automated method of measuring the PCV and HB has been designed and implemented. In order to validate the implemented system, the blood sample were taken and tested from 6 subjects ( 3 males and 3 females) with different ages (average: $33.16 \pm 7.19$ years). The comparison has been made between the results of the proposed automated system and conventional method of calculating the PCV. Table 1 shows the comparison between the readings of the automated and the conventional methods. The measurement of the conventional method had been done by a specialist physician in order to obtain an optimum reading and reduce the human error. It can be observed that the difference between the two readings is considerably small which is might be due to the subjective error. 


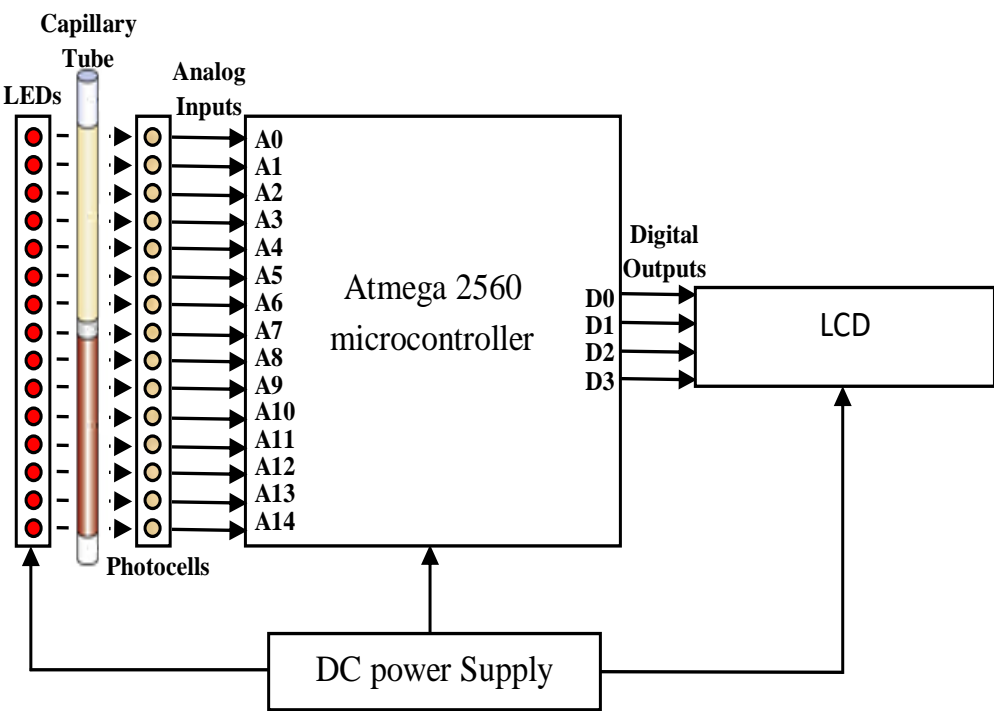

Figure 6. The schematic diagram of the proposed system

Table 1. The Compactions Between the Readings of the Automated and the Conventional Methods

\begin{tabular}{cccccc}
\hline Subject & Gender & Age (Years) & Automatic readings PCV\% & $\begin{array}{c}\text { Manual } \\
\text { Readings PCV \% }\end{array}$ & Difference \% \\
\hline S1 & Male & 35 & 40 & 38 & $2 \%$ \\
S2 & Male & 42 & 46.6 & 46 & $0.6 \%$ \\
S3 & Male & 25 & 46.5 & 46 & $0.5 \%$ \\
S4 & female & 35 & 38.7 & 38 & $0.7 \%$ \\
S5 & female & 38 & 36 & 36 & $0 \%$ \\
S6 & female & 24 & 40 & 40 & $0 \%$ \\
\hline
\end{tabular}

The effectiveness of the optical isolated material has also been studied. Figure 7 shows that the PCV readings of the automated system without the optical isolated material have a large variation comparing to the PCV reading of the automated system with the optical isolated material and the conventional method. This variation can be due to the interference and the scattering of the light which is lead to inaccurate evaluation of the PCV values. The statistically significant positive correlation $(r=0.9856, p=3.106 * 10-4)$ between the PCV readings of the automated system with the optical isolated material and the conventional method has been observed which indicated the accuracy and the precision of the proposed system. The comparison between the automated and the conventional methods is very important to insure the accuracy of the performance of the proposed method and eliminate the false positive readings of the PCV [26].

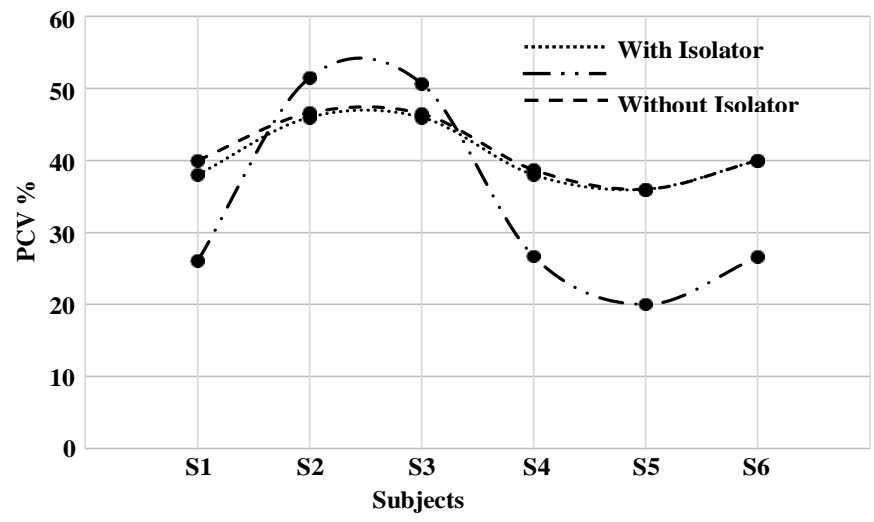

Figure 7. The PCV measurement using automated method with and without the isolator material as well as the conventional method 


\section{CONCLUSION}

Accurate evaluation of hematocrit of the patients with hematologic disorders is essential for the patients' safety. This study aimed to design, implement and evaluate the automated system for measuring the hematocrit of the centrifuged blood sample in a capillary tube. The statistically significant positive correlation was observed between the PCV readings of the proposed and conventional method. Using the isolator materials between the light source and photo detectors is necessary to reduce the false positive readings of the PCV. This paper can consequently be esteemed as an attempt to develop or to shadow a diagnostic model for an accurate, fast and cost-effective HCT measurement system. As a future work, the laser diode can be used in this system to increase the directivity of the light and the precision of the reading.

\section{REFERENCES}

[1] S. McKew, I. Bates, and E. Olayemi, "Hematologic Diseases," in Hunter's Tropical Medicine and Emerging Infectious Disease, Tenth Edit., Elsevier Inc., 2019, pp. 34-43.

[2] J. F. Friedman and K. Tolentino, "An update on anemia in less developed countries," Am. J. Trop. Med. Hyg., vol. 77, no. 1, pp. 44-51, 2007.

[3] J. A. H. Young, "Management of Infectious Diseases in Stem Cell Transplantation and Hematologic Malignancy," Infect. Dis. Clin. North Am., vol. 33, no. 2, pp. xiii-xv, 2019.

[4] P. L. Greenberg, V. Gordeuk, S. Issaragrisil, N. Siritanaratkul, S. Fucharoen, and R. C. Ribeiro, "Major hematologic diseases in the developing world- new aspects of diagnosis and management of thalassemia, malarial anemia, and acute leukemia.," Hematology, pp. 479-498, 2001.

[5] A. Tefferi, C. A. Hanson, and D. J. Inwards, "How to Interpret and Pursue an Abnormal Complete Blood Cell Count in Adults," Mayo Clin Proc., vol. 80, no. 7, pp. 923-936, 2005.

[6] R. A. McINROY, "A micro-haematocrit for determining the packed cell volume and haemoglobin concentration on capillary blood.," J. Clin. Pathol., vol. 7, no. 1, pp. 32-36, 1954.

[7] M. Venkata Dasu and P. Subbaiah, "Leukaemia Detection in Microscopic Imagery using Optimization Algorithm," Int. J. Eng. Technol., vol. 7, no. 2.24, pp. 299, 2018.

[8] G. Gebretsadkan, K. Tessema, H. Ambachew, and M. Birhaneselassie, "The Comparison between Microhematocrit and Automated Methods for Hematocrit Determination," Int J Blood Res Disord, vol. 2, pp. 1, 2015.

[9] R. Dey, K. Roy, D. Bhattacharjee, M. Nasipuri, and P. Ghosh, "An automated system for measuring hematocrit level of human blood from total RBC count," 2016 Int. Conf. Adv. Comput. Commun. Informatics, ICACCI 2016, pp. 2273-2279, 2016.

[10] P. S. Chari and S. Prasad, "Pilot Study on the Performance of a New System for Image Based Analysis of Peripheral Blood Smears on Normal Samples," Indian J. Hematol. Blood Transfus., vol. 34, no. 1, pp. 125-131, 2018.

[11] N. S. Fatonah, H. Tjandrasa, and C. Fatichah, "Automatic Leukemia Cell Counting using Iterative Distance Transform for Convex Sets,” Int. J. Electr. Comput. Eng., vol. 8, no. 3, pp. 1731-1740, 2018.

[12] R. Supriyanti, A. Chrisanty, Y. Ramadhani, and W. Siswandari, "Computer Aided Diagnosis for Screening the Shape and Size of Leukocyte Cell Nucleus based on Morphological Image," Int. J. Electr. Comput. Eng., vol. 8, no. 1, pp. 150-158, 2018.

[13] N. Chatap and S. Shibu, "Analysis of blood samples for counting leukemia cells using Support vector machine and nearest neighbour," IOSR J. Comput. Eng., vol. 16, no. 5, pp. 79-87, 2014.

[14] E. Hussein and A. Enein, "Clinical and Quality Evaluation of Red Blood Cell Units Collected Via Apheresis Versus Those Obtained Manually," Lab Med., vol. 45, no. 3, pp. 238-243, 2014.

[15] L. K. Goodhead and F. M. Macmillan, "Measuring osmosis and hemolysis of red blood cells," Adv Physiol Educ, vol. 41, pp. 298-305, 2019.

[16] B. J. Kim, Y. S. Lee, A. Zhbanov, and S. Yang, "A physiometer for simultaneous measurement of whole blood viscosity and its determinants: hematocrit and red blood cell deformability," Analyst, vol. 144, no. 9, pp. 3144-3157, 2019.

[17] M. M. A. Jamil, L. Oussama, W. M. Hafizah, M. H. A. Wahab, and M. F. Johan, "Computational Automated System for Red Blood Cell Detection and Segmentation". Elsevier Inc., 2019.

[18] A. ELEN and M. K. TURAN, "Classifying White Blood Cells Using Machine Learning Algorithms," Uluslararasl Muhendis. Arastirma ve Gelistirme Derg., vol. 11, no. 1, pp. 141-152, 2019.

[19] W. A. Salah and B. A. Zneid, "Evolution of microcontroller-based remote monitoring system applications," Int. J. Electr. Comput. Eng., vol. 9, no. 4, pp. 2354-2364, 2019.

[20] H. F. J. Baban and S. M. H. Yaseen, "Design and Implementation of PCV Device Based on Arduino Board," J. AlNahrain Univ., vol. 19, no. 4, pp. 16-20, 2016.

[21] H. H. Billett, "Hemoglobin and Hematocrit," in Clinical Methods: The History, Physical, and Laboratory Examinations., 3rd editio., H. J. Walker HK, Hall WD, Ed. Boston: Butterworths, 1990, pp. 718-719.

[22] J. V. Corbett and A. D. Banks, "Laboratory tests and diagnostic procedures: with nursing diagnoses". 2013.

[23] B. S. Bull, J. A. Koepke, E. Simson, and O. W. Van Assendelft, "Procedure for Determining Packed Cell Volume by the Microhematocrit Method; Approved Standard," in Clinical and labrotory Standards Institute, Third Edit., vol. 20, no. 18, 2001. 
[24] B. S. Bull, K. Fujimoto, B. Houwen, G. Klee, L. Van Hove, and O. W. Van Assendelft, "International Council for Standardization in Haematology ( ICSH ) Recommendations for 'Surrogate Reference' Method for the Packed Cell Volume Table of Contents," Lab. Hematol., vol. 9, pp. 1-9, 2003.

[25] A. Nayyar and V. Puri, "A Review of Arduino Board's, Liypad's and Arduino Shields," in 3rd International Conference on Computing for Sustainable Global Development (INDIACom), 2016, pp. 1485-1492.

[26] S. T. Avecilla et al., "Comparison of Manual Hematocrit Determinations vs Automated Methods for Hematopoietic Progenitor Cell Apheresis Products,” J Autism Dev Disord, vol. 47, no. 3, pp. 549-562, 2017.

\section{BIOGRAPHIES OF AUTHORS}

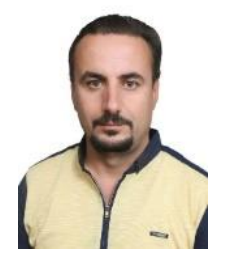

M. S. Of engineering (Electrical-computer and microelectronic system) University Teknologi Malaysia (UTM) Malaysia, 2011. B. Eng. in medical Instrumentation Technology Engineering 1999. Main responsibility: (work as a lecturer at Technical Engineering College, Northern Technical University.

Subjects of interest: Biomedical instrumentation, digital image processing, advance digital electronic, microcontroller.

$\mathrm{PhD}$ in Biomedical Engineering, University of Glasgow, Glasgow, United Kingdom, 2017. Technical Master Degree in Medical Instrumentation Technology Engineering, Technical Engineering College of Mosul, Mosul, IRAQ. 2006. B. Eng. In medical Instrumentation Technology Engineering 2002. Head of Medical Instrumentation Technology Engineering Department, Technical Engineering College of Mosul, Northern Technical University, Mosul, IRAQ. 2017-Now. Research Gate: https://www.researchgate.net/profile/Mohammed_Jarjees

Google Scholar: https://scholar.google.com/citations?authuser=2\&user=eErzW3UAAAAJ 